\title{
An in silico study of the influence of vessel wall deformation on neointimal hyperplasia progression in peripheral bypass grafts
}

Francesca Donadoni ${ }^{a}$, Mirko Bonfanti ${ }^{a, b}$, Cesar Pichardo-Almarza ${ }^{a}$, Shervanthi Homer Vanniasinkam ${ }^{\mathrm{a}, \mathrm{c}, \mathrm{d}}$, Alan Dardik ${ }^{\mathrm{e}, \mathrm{f}}$, Vanessa Díaz-Zuccarini ${ }^{\mathrm{a}, \mathrm{b}}$

a Department of Mechanical Engineering, University College London, WC1E 7JE, UK ${ }^{b}$ Wellcome/EPSRC Centre for Interventional and Surgical Sciences (WEISS), Department of Medical Physics and Biomedical Engineering, University College London, W1W 7TS, UK ' Leeds Teaching Hospitals NHS Trust, LS1 3EX, UK

${ }^{\mathrm{d}}$ Division of Surgery, University of Warwick, Warwick, UK

'The Department of Surgery, Yale University School of Medicine, New Haven, CT, USA

${ }^{f}$ Veteran Affairs Connecticut Healthcare System, West Haven, CT, USA

\section{Corresponding Authors:}

Dr Vanessa Díaz-Zuccarini

UCL Mechanical Engineering

University College London

Torrington Place, London WC1E 7JE, UK

Phone: +44 (0)20 76793928

Fax: $+44(0) 2073880180$

Electronic mail: v.diaz@ucl.ac.uk 


\section{Abstract}

Neointimal hyperplasia (NIH) is a major obstacle to graft patency in the peripheral arteries. A complex interaction of biomechanical factors contribute to NIH development and progression, and although haemodynamic markers such as wall shear stress have been linked to the disease, these have so far been insufficient to fully capture its behaviour. Using a computational model linking computational fluid dynamics (CFD) simulations of blood flow with a biochemical model representing NIH growth mechanisms, we analyse the effect of compliance mismatch, due to the presence of surgical stitches and/or to the change in distensibility between artery and vein graft, on the haemodynamics in the lumen and, subsequently, on NIH progression. The model enabled to simulate NIH at proximal and distal anastomoses of three patient-specific end-to-side saphenous vein grafts under two compliance-mismatch configurations, and a rigid wall case for comparison, obtaining values of stenosis similar to those observed in the computed tomography (CT) scans. The maximum difference in time-averaged wall shear stress between the rigid and compliant models was 3.4 Pa, and differences in estimation of NIH progression were only observed in one patient. The impact of compliance on the haemodynamic-driven development of NIH was small in the patient-specific cases considered.

Keywords: neointimal hyperplasia, compliance mismatch, multi-scale modelling, computational fluid dynamics, moving boundary method 


\section{Introduction}

Neointimal hyperplasia (NIH) commonly occurs as a side effect subsequently to peripheral bypass surgery, causing a high proportion of grafts to fail. The condition develops in the intimal layer of the arterial tissue due to complex interactions of the cellular components with the new environment (the graft), and, amongst multiple other factors, it is also influenced by haemodynamic effects in the vessel lumen [1]. More specifically, in peripheral venous bypass grafts, NIH often occurs at the proximal and distal anastomosis [2], where local flow patterns are affected by a number of factors, including the chosen surgical technique and compliance mismatch between venous and arterial wall.

Numerous computational fluid dynamics (CFD) studies have simulated the behaviour of peripheral bypass grafts with the assumption of rigid artery walls [3,4], but so far the effect of movement of the arterial wall in peripheral arteries has not been considered. Moving arterial walls have been shown to affect the distribution of wall shear stress in previous studies of aortic haemodynamics [5-8]. Although pressure values in the aorta are higher compared to the peripheral arteries, and the haemodynamic environment differs, the blood flow in major peripheral arteries, such as the femoral artery, might also be affected by the assumption of rigid walls.

In addition, the issue of compliance mismatch, an aspect widely documented to impact graft patency, can only be analysed by accounting for wall movement in the CFD analysis. So far, compliance mismatch has been accounted for mostly in computational studies on artificial stents $[9,10]$, and in coronary bypasses [11-13]. Arteriovenous grafts in the peripheral arteries can also present compliance mismatch due to the different mechanical properties of the artery and the venous graft. In fact, while veins are more compliant than arteries at low venous pressures, their mechanical behaviour drastically changes once exposed to the arterial environment. As shown by Stooker et al. [14], saphenous vein grafts subjected to high pressures (>75 $\mathrm{mmHg}$ ) lose their distensibility and behave as rigid tubes. On the contrary, arteries remain relatively distensible in the high-pressure range. With time, it is expected that a successful graft will arterialise with a corresponding change in mechanical properties [15]. Apart from these, another source of mismatch is due to the suture material of the anastomoses, which is often rigid and non-absorbable.

By altering the local pressure and velocity gradients, discontinuities in the compliance of the arteriovenous graft may cause critical changes in local haemodynamics [9], e.g., changes in wall shear stress (WSS) and oscillatory shear index (OSI) [16]. Numerous studies to date have looked at the effect of compliance mismatch in artificial stents $[9,17,18]$, using computational models to investigate the importance of different material properties in stent design [19], as well as to assess their performance under different haemodynamic conditions [20,21]. Computational research has also focused extensively on coronary grafts [11-13,22]. However, the effect of compliance mismatch in saphenous vein grafts, specifically in the peripheral 
arteries, has been investigated mainly through the use of animal models [23], with only one study where a computational model of a femoropopliteal bypass accounting for compliance mismatch between the artery and the graft showed much less significant displacements in comparison to larger arteries such as the aorta [24]. This makes an excellent case for the use of computational models to explore the role of compliance mismatch in human peripheral vein-grafts.

In this paper, we provide a quantifiable measure of the haemodynamic effect of changes in compliance in vein grafts, shedding some light on the likelihood of NIH progression inside the graft. Using in silico techniques, we aim at estimating the influence of vessel wall deformation on $\mathrm{NIH}$ growth in three individual patients who underwent saphenous vein graft surgery. For each of the patient-specific cases, we considered two configurations:

(i) post-operative scenario: compliant arterial segments and rigid venous graft;

(ii) post-operative after arterialisation: compliant arteriovenous graft.

In both cases, the presence of quasi-rigid surgical stitches was accounted for. The obtained results were compared against the outcomes of a rigid wall model developed in previous work [25].

\section{Methods}

Three patient-specific peripheral bypass grafts were analysed in silico and compared with previous work [25]. Based on the established hypothesis that abnormal values of wall shear stress alter the behaviour of vascular tissue [26], we developed a multi-scale simulation framework that combines haemodynamic and biological mechanisms to estimate $\mathrm{NIH}$ progression.

A key development of this work is the implementation of compliant vessel walls, using a moving boundary method, described in the following section. Other simulation conditions are analogous to those described in our previous publication [25] and omitted here for brevity. A summary of the simulation framework with its application to the patient-specific cases presented in this analysis is outlined in Figure 1.

\section{Moving Boundary Method}

The moving boundary method implemented here overcomes the limitations of using rigid walls in cardiovascular simulations, which is well-known to impact significantly CFD analyses in aortic studies [7] while simultaneously avoiding the complexity of a full fluid-structure interaction approach. Details on the implementation of the moving boundary method are reported in the study by Bonfanti et al. [6], and summarised here below. 
The displacement of the vessel wall follows the local surface-normal direction and is assumed linearly related to the local blood pressure. The displacement $\delta_{i}[\mathrm{~m}]$ of each mesh node $i$ on the vessel wall at each time-step is prescribed by Eq. (1):

$$
\delta_{i}=\frac{p_{i}-p_{\text {ext }}}{K_{i}} \mathbf{n}_{i}
$$

where $p_{i}[\mathrm{~Pa}]$ is the pressure at each node, passed from the fluid solution to the displacement equation, $p_{\text {ext }}[\mathrm{Pa}]$ is the external pressure set as equal to the diastolic pressure, $K_{i}\left[\mathrm{~N} / \mathrm{m}^{3}\right]$ is the stiffness coefficient, and $\mathbf{n}_{\boldsymbol{i}}$ is the local unit normal vector in the outward direction.

The value of $K_{i}$ is calculated based on the local cross-sectional area (A) of the vessel as follows:

$$
K_{i}=\frac{2}{\mathcal{D}} \times \sqrt{\frac{\pi}{A}}
$$

where $\mathcal{D}$ is the distensibility, defined as the relative change of the vessel cross-sectional area during a cardiac cycle divided by the corresponding blood pressure change [27].

In order to stabilise the simulations, a stabilisation parameter (namely "Mass Flux Pressure Coefficient" in CFX, ranging from $3 \times 10^{-3}$ to $10^{-1}\left[\mathrm{~kg} /\left(\mathrm{s} \cdot \mathrm{m}^{2} \cdot \mathrm{Pa}\right)\right]$ based on the simulation) was applied at the moving wall boundaries. A no-slip condition is applied to the vessel wall (i.e. the fluid velocity is equal to the wall velocity, which derives from the displacement calculated via Eq. (1)). The mesh displacement equations are solved so as to obtain an implicit two-way coupling between the mesh motion and fluid dynamics.

\section{Computational fluid dynamics}

The CFD simulations were implemented and solved with the Finite Volume Methodcommercial solver ANSYS-CFX v18 (ANSYS Inc., PA, USA), following the methodology reported in our previous publication [25]. In the ANSYS-CFX solver, the Navier-Stokes equations are spatially and temporally discretized with a high-resolution advection scheme [28] and a second-order implicit backward Euler scheme, respectively, using a uniform time step of 2.5 ms, good enough for time-step size-independent results [25]. ANSYS-CFX uses a coupled approach which solves the discretised conservation equations for the velocity $(u, v, w)$ and pressure (p) as a single system. An Incomplete Lower Upper factorisation technique with Additive Correction Algebraic Multigrid [29] is used to solve the system of linearised equations. A mesh-sensitivity analysis was performed for all three geometries to ensure independency of the results from the mesh size. The CFD simulations were carried out until reaching the periodic steady-state, which took 4-5 cycles. The results obtained in the last cycle were used for the analysis. The fluid domain was discretised with a hybrid mesh consisting of tetrahedral elements in the core region and 7 prismatic layers at the wall boundaries. In case of moving domains, CFX uses an ALE formulation of the fluid-dynamic conservation equations to account for the deformation in time of the control volumes [30]. 
Previous findings [31] highlighted the importance of considering the non-Newtonian behaviour of viscosity, but showed no difference in terms of NIH growth estimate at the proximal and distal anastomosis for a parabolic or plug flow inflow condition, hence, a plug flow condition was used at the inlet, and a non-Newtonian Carreau-Yasuda model of viscosity [32] was used. Patient-specific doppler ultrasound-derived flowrate waves $\left(Q_{1 N, 1}, Q_{1 N, 2}, Q_{1 N, 3}\right)$ were applied at the inlets as shown in Figure 2.

3-element RCR Windkessel models were applied at the model outlets. For efficient parameter tuning, we used, as a first approximation, an analog lumped-parameter model of the system simulated in 20-sim (v4.6, Controllab Products, B.V., The Netherlands). An optimisation method (Broyden Fletcher Goldfarb Shanno (BGFS), a gradient-descent algorithm based on the quasi-Newton method implemented in 20-Sim) was used to find the optimal WK3 parameters, among a range of possible values, which allowed obtaining the minimum difference between the integrals of the model and the target (obtained from Doppler data) flow rate curves. The values of the RCR parameters, reported in Table 1, were used in the 3D simulations. The new set of boundary conditions, with the corresponding diagram is shown in Figure 2.

\section{Neointimal Hyperplasia Growth Model}

The CFD computational simulations allow obtaining haemodynamic metrics linked to NIH growth. Specifically, in this work the Highly Oscillatory Low Magnitude Shear (HOLMES) index is used, defined by Eq. (3) following Alimohammadi et al. [33]:

$$
\text { HOLMES }=\text { TAWSS } \cdot(0.5-\text { OSI })
$$

where TAWSS = time-averaged wall shear stress, and OSI = oscillatory shear index. The values of the index at each node of the computational mesh are the input to the mathematical model of NIH progression [25]. This model is developed using ordinary differential equations solved in Matlab (The MathWorks, Inc., MA, USA) and takes into account the production of smooth muscle cells, collagen and growth factors, thus establishing a cause-effect relationship between the biological transformation inside the graft and the mechanical stimuli on the vessel wall, due to blood flow patterns. Once obtained the simulation results, values of the HOLMES index were extracted, and the biochemical model was run to estimate NIH growth. The framework used in this analysis is multi-scale, involving mechanisms at different spatial (from the model of cells to the 3D artery model), and temporal (time-scale of days in the cellular model and seconds in the CFD simulations) scales. The results from CFD simulations are used to compute occlusion by informing the biochemical model using a wall shear stress index, in this case HOLMES, for which results are available at each node in the mesh, and are assumed to be descriptive of the flow for the duration of disease development. The calculations for lumen occlusion were performed consistently with what was described in our previous publication [25], using Ansys CFD-Post (ANSYS, Inc.) for visualisation and measurement of simulated growth. 


\section{Simulation scenarios}

Two scenarios were considered and simulated for the three patients (Fig. 1):

1. Post-operative, not Arterialised (PO-NA): in the first set of simulations, we consider the conditions immediately after surgery and before the process of arterialisation occurs. The saphenous vein extracted from the venous environment (where it is highly compliant, at low venous pressure) and placed in the new arterial environment, becomes almost completely rigid to sustain the higher arterial pressure [34]. Hence, a distensibility $\mathcal{D}=$ $4.4 \cdot 10^{-3} \mathrm{kPa}^{-1}$ was assigned to the arterial segments, as reported by the existing literature on femoral artery tissue properties in patients with a cardiovascular event [35], while a rigid wall was assumed for the venous graft. A distensibility $\mathcal{D}=1.1 \cdot 10^{-3} \mathrm{kPa}^{-1}$ was assigned to the wall regions corresponding to the suture stitches, in order to simulate the mechanical properties of non-absorbable filaments of Prolene ${ }^{\circledR}$ [36]. The area covered by the stitches was assumed to be a $2 \mathrm{~mm}$ thick ring along the wall.

2. Post-opertive Arterialised (PO-A): in this scenario, the venous graft was assumed to have fully undergone the process of arterial adaptation [15], and thus to have obtained the same value of distensibility ( $\left.\mathcal{D}=4.4 \cdot 10^{-3} \mathrm{kPa}^{-1}\right)$ of the native femoral artery. The presence of the surgical suture was considered also in this scenario.

The results obtained from the PO-NA and PO-A simulations were compared with the results obtained from the analysis assuming rigid walls $(R)$.

\section{Results}

For each scenario (i.e. PO-NA, PO-A and R), mass flow rates at the main outlet were compared to ensure that similarity of the curves was achieved at the boundaries. As a result, velocities were similar in all three wall model cases. In Patient 1, velocity values averaged over the main outlet and over time are $0.800 \mathrm{~m} / \mathrm{s}, 0.816 \mathrm{~m} / \mathrm{s}$ and $0.810 \mathrm{~m} / \mathrm{s}$ in the R, PO-A and PO-NA cases, respectively (2.0\% difference between $R$ and PO-A cases and $1.2 \%$ difference between $R$ and PO-NA case). In Patient 2, the same measurements are respectively $0.107 \mathrm{~m} / \mathrm{s}, 0.111 \mathrm{~m} / \mathrm{s}$, $0.110 \mathrm{~m} / \mathrm{s}$ (difference of $3.7 \%$ between $\mathrm{R}$ and PO-A case and $2.8 \%$ between R and PO-NA case). In Patient 3 there were no differences measured. For comparison, results for flow rate and pressure profiles at the inlets and outlets, which were within the physiological range, are shown in Figure 3.

Analysis of velocity at peak systole shows areas of stagnation in zones where curvature is high; in other words, where the centreline of the vessel deviates highly from a straight line, particularly in the lower half of the bypass in Patients 2 and 3. Stagnation in these areas contributes to lower wall shear stress values.

Although displacements of the arterial wall were on average small, up to a maximum of 2.4 $x 10^{-2} \mathrm{~mm}$, they resulted in slightly different haemodynamic behaviour, especially close to the walls. 
A closer look at the velocity vectors at peak systole on the cross-sectional planes corresponding to the proximal and distal anastomosis (Supplementary Figure 1), explains the differences between the three cases. As shown in Supplementary Figure 1, the largest differences in velocity are found close to the wall, which translates in differences in wall shear stress. The largest differences are found between the $\mathrm{R}$ and PO cases, rather then between PO-A and PO-NA. For comparison, area-averaged velocities at peak systole at the proximal anastomosis, mid-graft and distal anastomosis for Patient 2 are reported in Table 2 (values for patients 1 and 3 are reported in Supplementary Tables 1 and 2).

For further evaluation of the flow characteristics in the lumen, contour maps of TAWSS and HOLMES were obtained. Differences in shear stress indices were largest between the $R$ and PO-A cases for all patients, with smaller differences between the R and PO-NA cases, where the venous graft had a rigid behaviour. The maximum difference in TAWSS amongst the different wall models is found in Patient 2 between the R and PO-A models at 3.4 Pa. Given the impact of low values of shear stress on NIH progression, a difference of 3.4 Pa could significantly change the model results, however in this case it only affected a very small area, while in the rest of the geometry the differences were significantly lower. With regard to the HOLMES index, the differences, although lower than for TAWSS, are still significant for the model, reaching a maximum value of $1.7 \mathrm{~Pa}$ (Patient 2) which may impact simulation outcomes in terms of NIH growth, as the computational framework assumes a value lower than 0.5 Pa to be the threshold for the onset of the disease [25]. The overall difference in HOLMES (difference of the HOLMES value averaged along the wall) was highest in patient 2 (1.5 Pa average difference against 0.1 and 0.07 in Patients 1 and 3), which also had the highest maximum difference. However, Patient 3 presented higher differences at the proximal and distal anastomoses, the areas more critical to NIH development (Figure 4).

After obtaining values for the haemodynamic variables of interest, the HOLMES index was used as an input to the biochemical model of NIH to estimate growth. Proportions of occluded lumen areas were obtained for each simulation case in the three patients at the two locations of most severe NIH development (corresponding to the proximal and distal anastomoses). For all patients, PO-NA and PO-A scenarios predicted equivalent results (maximum difference of only $1 \%$ at the proximal anastomosis for Patient 3 ). In Patient 1 and Patient 2, rigid-wall (R) and moving-wall scenarios (PO-A and PO-NA) gave similar occlusion results (maximum difference of $1 \%$ at the distal anastomosis for Patient 1 , and of $3 \%$ at the proximal anastomosis for Patient 2). The highest difference was observed for Patient 3 at the proximal anastomosis, with a predicted occlusion of $31 \%$ for the PO-A scenario and of $38 \%$ for the rigid model. Final NIH occlusion results from the simulations are reported in Supplementary Figure 2 and summarized in Table 3. 


\section{Discussion}

This study addresses the current need to expand simulation models of peripheral bypass grafts beyond haemodynamic analysis in rigid wall models. Similarly to the research that has been recently conducted for the understanding of disturbances in the flow due to moving walls in coronary artery grafts $[37,11]$, we aim at understanding the impact of graft-artery compliance mismatch on disease progression in peripheral vascular grafts.

The simulations presented in this analysis allowed to measure important haemodynamic indicators such as TAWSS and OSI while accounting for walls movement. This is important in the context of NIH in peripheral vascular grafts as the current research has not yet defined quantitatively the effect of compliance mismatch on the haemodynamics in this particular type of graft, although a first study from Colciago et al. showed a much reduced effect of displacements in comparison with the aorta [24]. Our model, besides providing a detailed study of the blood flow within the grafts, allows the simulation of NIH progression via a multiscale simulation framework based on haemodynamic shear-stress based markers. Our analysis showed that in three case studies of peripheral arterial bypasses, the effect of moving walls on the haemodynamics has only a small impact on NIH development.

The framework used in this analysis is based on the detailed simulation of the haemodynamic conditions inside vein-grafts coupled with the main biological mechanisms involved in neointimal growth [25]. Results show that haemodynamic-driven $\mathrm{NIH}$ progression was affected by compliance mismatch only in one patient out of three, where occlusion of the bypass in the PO-A and PO-NA cases showed a small percentage difference compared to the rigid wall case (we consider as a source of compliance mismatch, both the different compliance between the native artery and the venous graft, and the presence of nondistensible surgical stitches). In this patient (Patient 3) small differences of $7 \%$ and $2 \%$ (at the proximal and distal anastomosis, respectively) were present, indicating that the effect of compliance on predicted values of NIH growth might be different in certain haemodynamic conditions and for certain graft geometries. Although further studies on larger cohorts of patients should be performed to assess the impact that compliance mismatch could have on $\mathrm{NIH}$ development in different cases, our initial analysis only showed a small effect and this represents an interesting contribution to the literature.

The analysis also confirmed the importance of using a composite index, such as HOLMES, in cardiovascular simulations evaluating haemodynamic-driven disease progression, as previously shown by predictive models of atherosclerotic plaque formation and progression [38-40]. As shown in Figures 4 and 5, small differences in the HOLMES index exist between the $\mathrm{R}$ and PO models, which are reflected on the occlusion simulations. Albeit small, these differences have an impact on the simulation results, and could not have been captured using a single index alone (e.g. TAWSS or OSI). At the proximal anastomosis of Patient 3, a lower average value of HOLMES in the rigid wall case resulted in a higher estimated occluded cross 
section of the lumen, while the opposite is valid at the distal anastomosis. As shown in Figure 5 , this difference in behaviour between the two areas can be explained by examining more closely the relationship between the components of HOLMES. The figure shows plots of the differences in values of the two shear stress indices, TAWSS and OSI, by means of which HOLMES is calculated. While the behaviour of TAWSS remains similar at the proximal and distal anastomosis, with lower values in the R case, for OSI the difference in values at the proximal anastomosis is very close to 0 (white correspond to zero in the map), and at the distal anastomosis the difference tends to be negative, meaning the OSI in the moving wall simulations is higher, which contributes to lowering the overall value of HOLMES.

Our study considered grafts that used end-to-side anastomotic techniques. Although previous numerical [13] and animal studies [41] showed a small impact of compliance mismatch in end-to-end grafts and a more significant impact in the end-to-side configuration, the debate on which technique minimizes NIH progression is still ongoing, with in vivo studies showing comparable patency for both configurations [40,41]. Using our current framework, studies controlling for different anastomotic techniques, such as end-to-end versus end-toside anastomosis, could be performed to quantify the effects of the two techniques on $\mathrm{NIH}$ development. The advantage of the computational framework is the possibility to run multiple simulations controlling for different conditions in a comparatively shorter time, which in this case can be used to quantify the effect of compliance mismatch on different types of graft, anastomosis techniques, and physiological parameters.

The analysis has highlighted how wall motion impacted the haemodynamics in the peripheral grafts analysed at key locations of NIH development (proximal and distal anastomosis) and along the graft. With saphenous vein grafts still failing at severely high rates, it is important to understand and quantify the mechanisms beyond graft failure, using patient-specific models which can be tuned to specific conditions to provide testing frameworks for targeted clinical questions.

\section{Limitations}

In this specific case, as compliance data was only available for a generic patient and not for each of the unique cases analysed, we considered two hypothetical cases, one in which compliance was uniform along the graft and artery with a change only at the location of surgical stitches, and one which considered the graft not to be yet arterialised, with a mismatch in compliance between vein graft and arterial wall. This limits the use of this study in choosing the most suitable arterial wall model to be used in the case of peripheral bypass grafts, only allowing to measure and assess the difference in results in terms of three benchmark values of distensibility. In addition, limitations linked to the structure of the model are present, for instance the simplification of the process of NIH remodeling as a response to mechanical/haemodynamic stimuli and a limited selection of cellular mechanisms, neglecting secondary mechanisms that impact disease progression. Further biochemical species to include would be, for instance, growth factors such as vascular endothelial growth factor 
(VEGF), and basic fibroblastic growth factor (bFGF), extracellular matrix components and endothelial cells, which have been linked to NIH [15]. Phenotypic traits and co-morbidities also have an effect on disease progression, and their addition to the model might enhance the analysis towards a better patient-stratification. Finally, it is worth noting that although our study does not show a significant impact of compliance mismatch on the haemodynamicdriven progression of $\mathrm{NIH}$, arterial stiffness is closely linked to hypertension [44], a risk factor for other cardiovascular complications, and stiffer vascular conduits might have an adverse effect on patency through other biological mechanisms. For example, it is known that hypertension induces abnormalities to the intimal layer structure, that in turn can affect the underlaying media leading to smooth muscle cells necrosis and elastic fibers fibrosis [43].

\section{Conclusion}

This study shows the applicability of a multi-scale, patient specific model of NIH as a tool to measure haemodynamic effects on the disease progression, as well as a way to test different modelling approaches. A model of arterial wall motion has been tested on three patientspecific geometries of peripheral end-to-side, saphenous vein grafts, and two different scenarios involving compliance mismatch at the anastomoses were studied and compared against rigid-wall simulations.

The main conclusion is that differences in haemodynamics results had in most cases minimal impact on the NIH occlusion simulations, suggesting that a compliance mismatch in peripheral bypass graft, either due to the presence of surgical stitches or to the change in distensibility between artery and vein, has small impact on the haemodynamic-driven development of $\mathrm{NIH}$.

\section{Competing interests}

The authors declare no conflict of interest.

\section{Ethical approval}

The study was ethically approved from the institutional human investigation committee at Veteran Affairs Connecticut Healthcare System, West Haven, CT, USA (approval number: AD0009). The patients signed the appropriate consent form.

\section{Acknowledgements}

This project was supported by the Engineering and Physical Sciences Research Council (EPSRC) through the Doctoral Training Programme "Healthcare Engineering for an Ageing Population"; the resources and the use of facilities at the Veterans Affairs Connecticut Healthcare System (West Haven, CT); the European Union's Horizon 2020 research and innovation programme (Marie Sklodowska-Curie GA No. 642612, www.vph-case.eu); the Wellcome/EPSRC Centre 
for Interventional and Surgical Sciences (WEISS) (203145Z/16/Z); and the Leverhulme Trust (Senior Research Fellowship No. RF-2015-482).

\section{References}

[1] Model LS, Dardik A. Neointimal Hyperplasia : Basic Considerations 2012.

[2] Perktold K, Leuprecht A, Prosi M, Berk T, Czerny M, Trubel W, et al. Fluid Dynamics, Wall Mechanics, and Oxygen Transfer in Peripheral Bypass Anastomoses. Ann Biomed Eng 2002;30:447-60. doi:10.1114/1.1477445.

[3] Tarbell JM, Shi ZD, Dunn J, Jo H. Fluid Mechanics, Arterial Disease, and Gene Expression. Annu Rev Fluid Mech 2014;46:591-614. doi:10.1146/annurev-fluid-010313-141309.

[4] Murphy J, Boyle F. Predicting neointimal hyperplasia in stented arteries using time-dependant computational fluid dynamics: A review. Comput Biol Med 2010;40:408-18.

doi:10.1016/j.compbiomed.2010.02.005.

[5] Bonfanti M, Balabani S, Greenwood JP, Puppala S, Homer-Vanniasinkam S, Díaz-Zuccarini V. Computational tools for clinical support: a multi-scale compliant model for haemodynamic simulations in an aortic dissection based on multi-modal imaging data. J R Soc Interface 2017;14:20170632. doi:10.1098/rsif.2017.0632.

[6] Bonfanti M, Balabani S, Alimohammadi M, Agu O, Homer-Vanniasinkam S, Díaz-Zuccarini V. A simplified method to account for wall motion in patient-specific blood flow simulations of aortic dissection: Comparison with fluid-structure interaction. Med Eng Phys 2018;58:72-9. doi:10.1016/j.medengphy.2018.04.014.

[7] Alimohammadi M, Sherwood JM, Karimpour M, Agu O, Balabani S, Díaz-Zuccarini V. Aortic dissection simulation models for clinical support: fluid-structure interaction vs. rigid wall models. BioMedical Eng OnLine 2015;14. doi:10.1186/s12938-015-0032-6.

[8] Reymond P, Crosetto P, Deparis S, Quarteroni A, Stergiopulos N. Physiological simulation of blood flow in the aorta: Comparison of hemodynamic indices as predicted by 3-D FSI, 3-D rigid wall and 1-D models. Med Eng Phys 2013;35:784-91. doi:10.1016/ j.medengphy.2012.08.009.

[9] Selvarasu NKC, Tafti DK, Vlachos PP. Hydrodynamic Effects of Compliance Mismatch in Stented Arteries. J Biomech Eng 2011;133:21008. doi:10.1115/1.4003319.

[10] Nappi F, Carotenuto AR, Cutolo A, Fouret P, Acar C, Chachques JC, et al. Compliance mismatch and compressive wall stresses drive anomalous remodelling of pulmonary trunks reinforced with Dacron grafts. J Mech Behav Biomed Mater 2016;63:287-302. doi:10.1016/J.JMBBM.2016.06.023.

[11] Guerciotti B, Vergara C, Ippolito S, Quarteroni A, Antona C, Scrofani R. A computational fluidstructure interaction analysis of coronary Y-grafts. Med Eng Phys 2017;47:117-27. doi:10.1016/ j.medengphy.2017.05.008.

[12] Ramachandra AB, Kahn AM, Marsden AL. Patient-Specific Simulations Reveal Significant Differences in Mechanical Stimuli in Venous and Arterial Coronary Grafts. J Cardiovasc Transl Res 2016;9:279-90. doi:10.1007/s12265-016-9706-0.

[13] Ballyk PD, Walsh C, Butany J, Ojha M. Compliance mismatch may promote graft-artery intimal hyperplasia by altering suture-line stresses. J Biomech 1997;31:229-37. doi:10.1016/S01973975(97)00111-5.

[14] Stooker W, Gök M, Sipkema P, Niessen HW., Baidoshvili A, Westerhof N, et al. Pressurediameter relationship in the human greater saphenous vein. Ann Thorac Surg 2003;76:1533-8. 
doi:10.1016/S0003-4975(03)00896-8.

[15] Muto A, Model L, Ziegler K, Eghbalieh SDD, Dardik A. Mechanisms of Vein Graft Adaptation to the Arterial Circulation. Circ J 2010;74:1501-12. doi:10.1253/circj.CJ-10-0495.

[16] Morris L, Stefanov F, McGloughlin T. Stent graft performance in the treatment of abdominal aortic aneurysms: The influence of compliance and geometry. J Biomech 2013;46:383-95. doi:10.1016/j.jbiomech.2012.11.026.

[17] Sheth V, Ritter A. Using Computational Fluid Dynamics Model to Predict Changes in Velocity properties in Stented Carotid Artery 2010.

[18] Coimbatore Selvarasu NK. Investigation of the Hemodynamics of Coronary Arteries - Effect of Stenting 2013.

[19] Qiao A, Zhang Z. Numerical Simulation of Vertebral Artery Stenosis Treated With Different Stents. J Biomech Eng 2014;136:41007. doi:10.1115/1.4026229.

[20] Kabinejadian F, Ghista DN. Compliant model of a coupled sequential coronary arterial bypass graft: Effects of vessel wall elasticity and non-Newtonian rheology on blood flow regime and hemodynamic parameters distribution. Med Eng Phys 2012;34:860-72. doi:10.1016/j.medengphy.2011.10.001.

[21] Taelman L, Bols J, Degroote J, Muthurangu V, Panzer J, Vierendeels J, et al. Differential impact of local stiffening and narrowing on hemodynamics in repaired aortic coarctation: an FSI study. Med Biol Eng Comput 2016;54:497-510. doi:10.1007/s11517-015-1336-1.

[22] Frauenfelder T, Boutsianis E, Schertler T, Husmann L, Leschka S, Poulikakos D, et al. Flow and wall shear stress in end-to-side and side-to-side anastomosis of venous coronary artery bypass grafts. Biomed Eng Online 2007;6:35. doi:10.1186/1475-925X-6-35.

[23] Chen HY, Sinha AK, Choy JS, Zheng H, Sturek M, Bigelow B, et al. Mis-sizing of stent promotes intimal hyperplasia: impact of endothelial shear and intramural stress. Am J Physiol Circ Physiol 2011;301:H2254-63. doi:10.1152/ajpheart.00240.2011.

[24] Colciago CM, Deparis S, Quarteroni A. Comparisons between reduced order models and full 3D models for fluid-structure interaction problems in haemodynamics. J Comput Appl Math 2014;265:120-38. doi:10.1016/j.cam.2013.09.049.

[25] Donadoni F, Pichardo-Almarza C, Bartlett M, Dardik A, Homer-Vanniasinkam S, Díaz-Zuccarini V. Patient-Specific, Multi-Scale Modeling of Neointimal Hyperplasia in Vein Grafts. Front Physiol 2017;8:226. doi:10.3389/fphys.2017.00226.

[26] Tarbell JM, Simon SI, Curry F-RE, Edu : Sisimon@ucdavis. Mechanosensing at the Vascular Interface HHS Public Access. Annu Rev Biomed Eng July 2014;11:505-32. doi:10.1146/annurevbioeng-071813-104908.

[27] Ganten M, Krautter U, Hosch W, Hansmann J, von Tengg-Kobligk H, Delorme S, et al. Age related changes of human aortic distensibility: evaluation with ECG-gated CT. Eur Radiol 2007;17:701-8. doi:10.1007/s00330-006-0309-z.

[28] Barth TJ, Jespersen DC. The design and application of upwind schemes on unstructured meshes 1989.

[29] Raw M. Robustness of coupled Algebraic Multigrid for the Navier-Stokes equations. 34th Aerosp. Sci. Meet. Exhib., Reston, Virigina: American Institute of Aeronautics and Astronautics; 1996. doi:10.2514/6.1996-297.

[30] ANSYS Inc. ANSYS-CFX Solver Theory Guide 2016.

[31] Donadoni F, Pichardo-Almarza C, Homer-Vanniasinkam S, Dardik A, Diaz V. Analysis of the Haemodynamic Factors Involved in Neointimal Hyperplasia Growth in Femoro-Popliteal Bypass 
Grafts Using Different Multi-scale, Patient-specific Modelling Approaches. Eur J Vasc Endovasc Surg 2018;56:e19. doi:10.1016/j.ejvs.2018.06.083.

[32] Gijsen FJH, Allanic E, van de Vosse FN, Janssen JD. The influence of the non-Newtonian properties of blood on the flow in large arteries: unsteady flow in a $90^{\circ}$ curved tube. J Biomech 1999;32:705-13. doi:10.1016/S0021-9290(99)00014-7.

[33] Alimohammadi M, Pichardo-Almarza C, Agu O, Díaz-Zuccarini V. Development of a PatientSpecific Multi-Scale Model to Understand Atherosclerosis and Calcification Locations: Comparison with In vivo Data in an Aortic Dissection. Front Physiol 2016;7. doi:10.3389/fphys.2016.00238.

[34] Dobrin PB, Littooy FN, Golan J, Blakeman B, Fareed J. Mechanical and histologic changes in canine vein grafts. J Surg Res 1988;44:259-65.

[35] van Sloten TT, Schram MT, van den Hurk K, Dekker JM, Nijpels G, Henry RMA, et al. Local Stiffness of the Carotid and Femoral Artery Is Associated With Incident Cardiovascular Events and All-Cause Mortality: The Hoorn Study. J Am Coll Cardiol 2014;63:1739-47. doi:10.1016/J.JACC.2013.12.041.

[36] Ulrich M, Staalsen N, Djurhuus CB, Christensen TD, Nygaard H, Hasenkam JM. In vivo analysis of dynamic tensile stresses at arterial end-to-end anastomoses. Influence of suture-line and graft on anastomotic biomechanics. Eur J Vasc Endovasc Surg 1999;18:515-22. doi:10.1053/ejvs.1999.0937.

[37] Ramachandra AB, Kahn AM, Marsden AL. Patient-Specific Simulations Reveal Significant Differences in Mechanical Stimuli in Venous and Arterial Coronary Grafts. J Cardiovasc Transl Res 2016;9:279-90. doi:10.1007/s12265-016-9706-0.

[38] Alimohammadi M, Pichardo-Almarza C, Agu O, Díaz-Zuccarini V. A multiscale modelling approach to understand atherosclerosis formation: A patient-specific case study in the aortic bifurcation. Proc Inst Mech Eng Part H J Eng Med 2017;231:378-90. doi:10.1177/0954411917697356.

[39] Díaz-Zuccarini V, Agu O, Tomaso G Di, Pichardo-Almarza C. Towards personalised management of atherosclerosis via computational models in vascular clinics: technology based on patientspecific simulation approach. Healthc Technol Lett 2014;1:13-8. doi:10.1049/htl.2013.0040.

[40] DiTomaso G, Díaz-Zuccarini V, Pichardo-Almarza C. A Multiscale Model of Atherosclerotic Plaque Formation at Its Early Stage. IEEE Trans Biomed Eng 2011;58:3460-3. doi:10.1109/tbme.2011.2165066.

[41] Sottiurai VS, Lim Sue S, Feinberg EL, Bringaze WL, Tran AT, Batson RC. Distal anastomotic intimal hyperplasia: Biogenesis and etiology. Eur J Vasc Surg 1988;2:245-56. doi:10.1016/S0950821X(88)80034-3.

[42] Schouten O, Hoedt MTC, Wittens CHA, Hop WCJ, van Sambeek MRHM, van Urk H, et al. End-toend Versus End-to-side Distal Anastomosis in Femoropopliteal Bypasses; Results of a Randomized Multicenter Trial. Eur J Vasc Endovasc Surg 2005;29:457-62. doi:10.1016/J.EJVS.2005.01.010.

[43] Hoedt M, How T, Poyck P, Wittens C. Why Patencies of Femoropopliteal Bypass Grafts with Distal End-to-End Anastomosis are Comparable with End-to-Side Anastomosis. Ann Thorac Cardiovasc Surg 2015;21:157-64. doi:10.5761/atcs.0a.14-00121.

[44] Cecelja M, Chowienczyk P. Role of arterial stiffness in cardiovascular disease. JRSM Cardiovasc Dis 2012;1. doi:10.1258/cvd.2012.012016. 


\section{Figures}

\begin{tabular}{|c|c|}
\hline $\begin{array}{l}\text { Clinical } \\
\text { Data }\end{array}$ & $\begin{array}{l}\text { - Doppler Ultrasound } \\
\text { - CT Scans }\end{array}$ \\
\hline Patient 1 & $\begin{array}{l}\text { - Femoro-distal bypass } \\
\text { - Length: } 92 \mathrm{~cm} \\
\text { - } ø_{\text {INLET }} 12.5 \mathrm{~mm}\end{array}$ \\
\hline Patient 2 & $\begin{array}{l}\text { - Fem-pop bypass } \\
\text { - Length: } 47 \mathrm{~cm} \\
\text { - } \emptyset_{\text {INLET: }} 10.0 \mathrm{~mm}\end{array}$ \\
\hline Patient 3 & $\begin{array}{l}\text { - Fem-pop bypass } \\
\text { - Length: } 23 \mathrm{~cm} \\
\text { - } \emptyset_{\text {INLEF }} 7.0 \mathrm{~mm}\end{array}$ \\
\hline
\end{tabular}

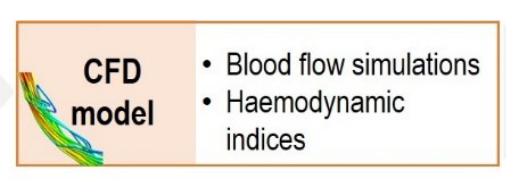

$\begin{array}{cl}\text { Rigid wall } & \text { - Artery: rigid } \\ (\mathrm{R}) & \text { - Stitches: rigid } \\ & \text { - Graft: rigid }\end{array}$

$\begin{array}{cl}\text { Post-op } & \text { - Artery: compliant } \\ \text { (PO-NA) } & \text { - Stitches: rigid } \\ & \\ \text { Post-op } & \text { - Artery: compliant } \\ \text { arterialised } & \text { - Stitches: rigid } \\ \text { (PO-A) } & \text { - Graft: compliant }\end{array}$
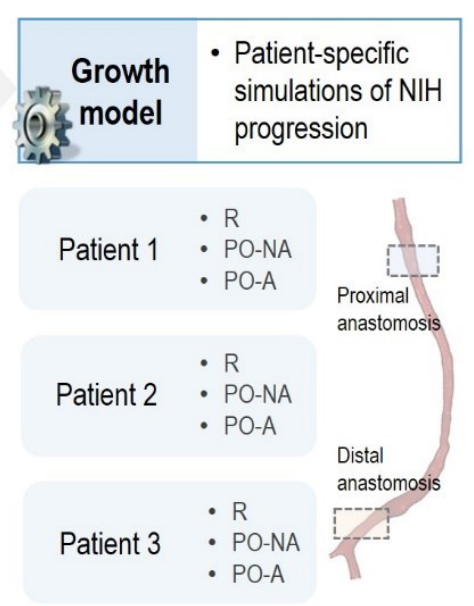

Figure 1: Summary of the simulation framework. Clinical data is acquired for three different patient-specific cases (Patients 1, 2 and 3). CFD analysis is performed considering three different scenarios: rigid-wall (R), Post-operative before graft arterialisation (PO-NA), Postoperative after graft arterialisation (PO-A). Finally, intimal hyperplasia growth is simulated for each patient in all three scenarios, and the results are compared at the locations of most severe hyperplasia (proximal and distal anastomosis). 


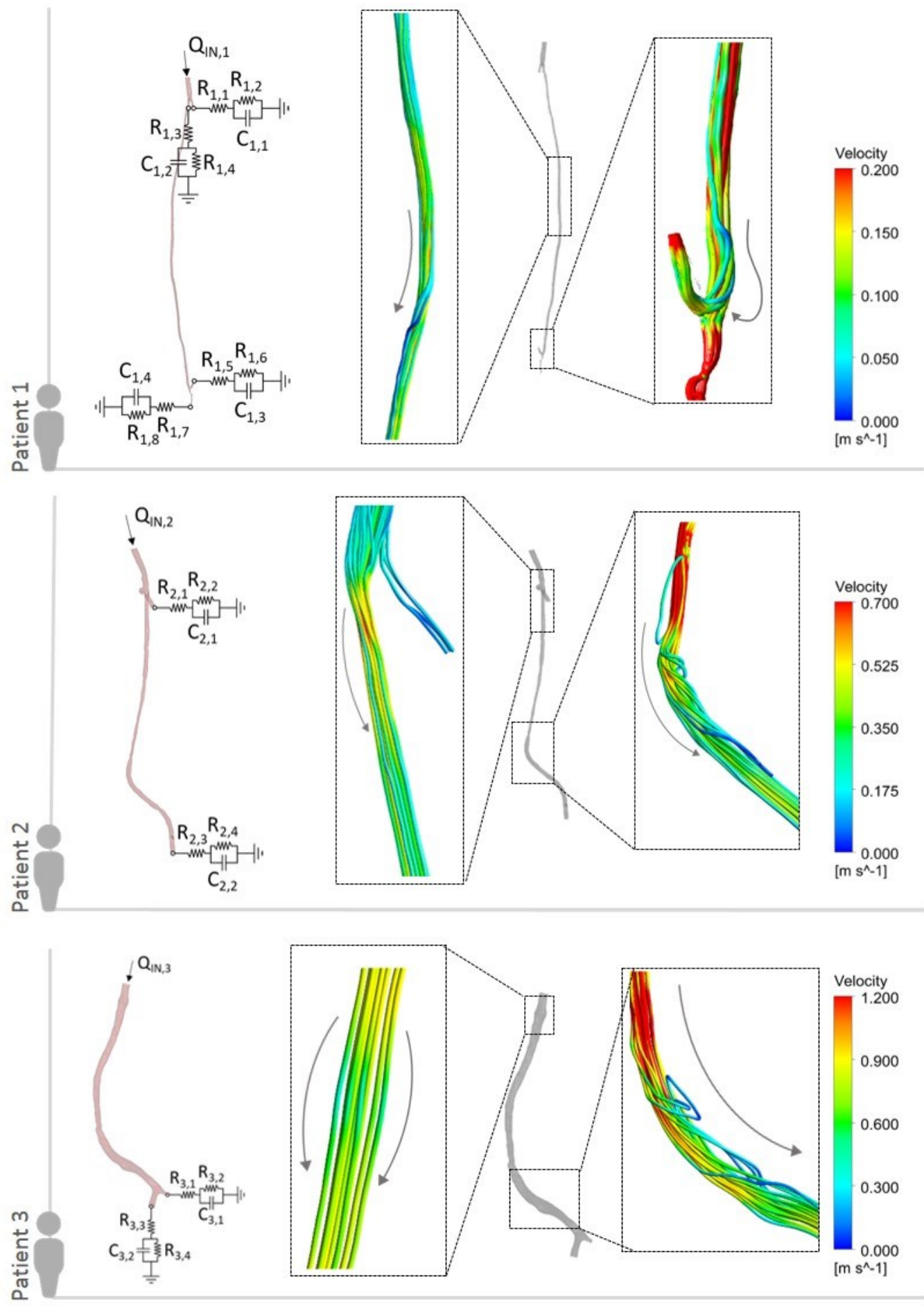

Figure 2: Left: Diagram of the patient-specific CFD models and boundary conditions for the three patients; Right: Velocity streamlines at peak systole computed for Patients 1, 2 and 3 in the post-operative-arterialised (PO-A) scenario. 

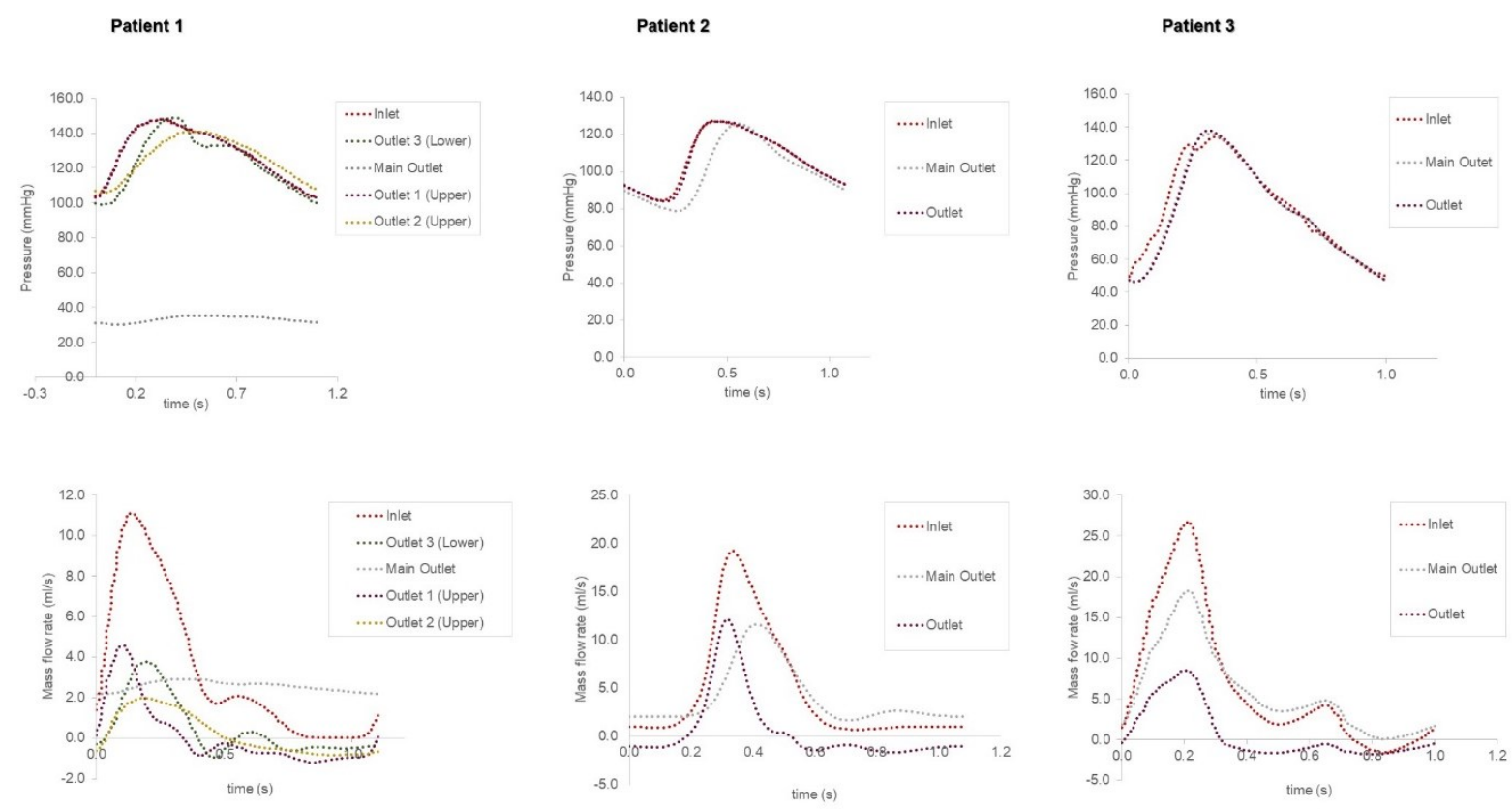

Figure 3: velocity and pressure profiles for patients 1, 2 and 3 in the $R$ case.
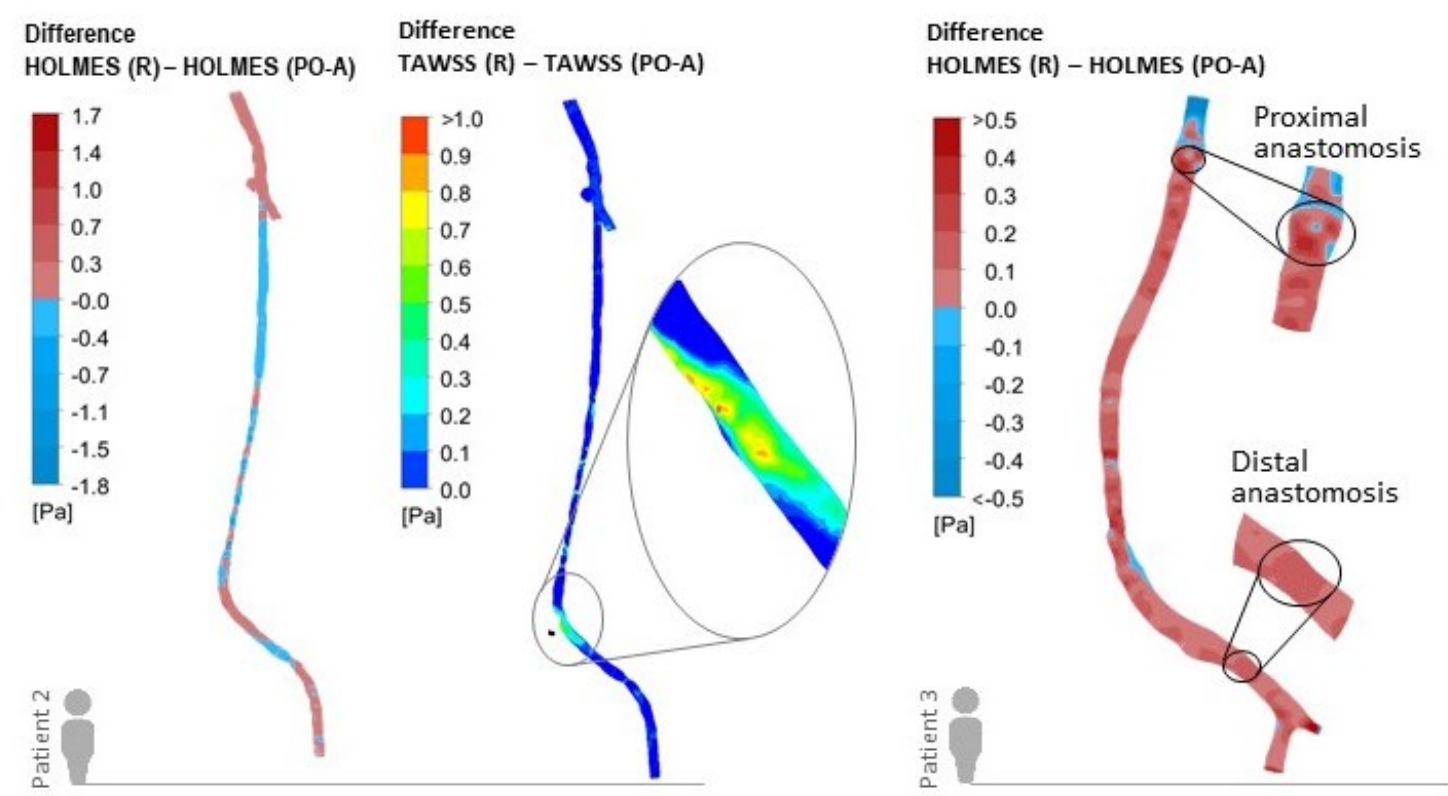

Figure 4: patient 2 presents the highest difference in HOLMES between $R$ and PO-A cases due to a high difference in TAWSS, however in patient 3 high differences are found at the critical locations for NIH development (proximal and distal anastomosis). Comparisons for all patients are given in the Supplementary Data. 

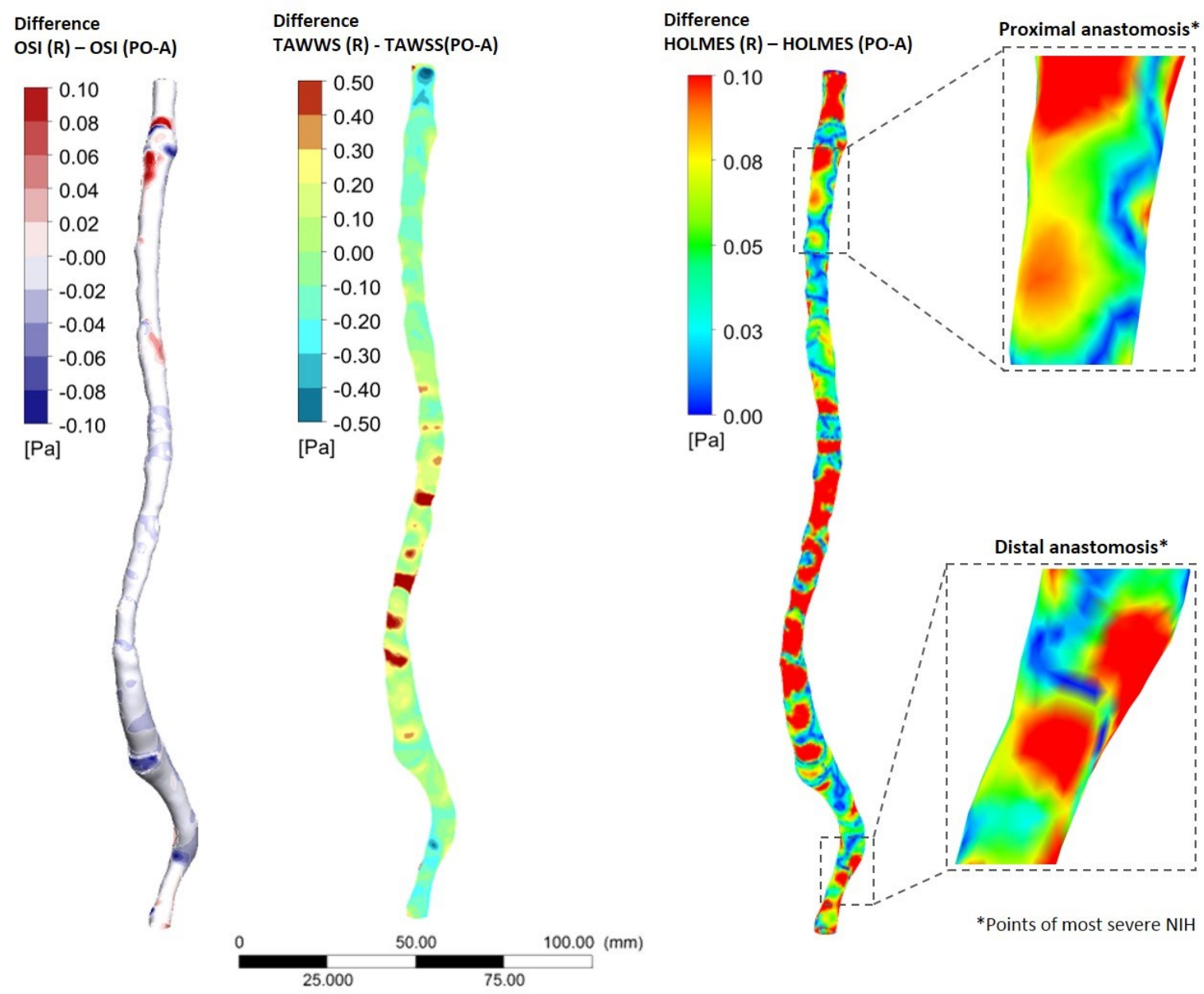

Figure 5: Differences in haemodynamic indices (TAWSS, OSI and HOLMES) between $R$ and POA scenarios for Patient 3, with areas of highest difference in HOLMES at the proximal and distal anastomosis shown in zoomed-in areas. The differences are computed by subtracting the values of the indices for the moving wall results from those for the rigid wall. In the TAWSS contour plot, at the proximal anastomosis the areas tend to the negative end of the scale (light blue), while at the distal anastomosis they are mostly positive (green to red), meaning TAWSS in the rigid wall case is higher, leading to a higher HOLMES, which results in a lower estimated occlusion. 


\section{Tables}

Table 1: Parameters of the 3-element Windkessel boundary conditions in the three patients. The first subscript refers to the patient (1,2 and 3).

\begin{tabular}{ccc}
\hline $\begin{array}{c}\text { Proximal resistance } \\
{\left[\mathbf{m m H g ~ s ~ m l}^{-1}\right]}\end{array}$ & $\begin{array}{c}\text { Distal Resistance } \\
{\left[\mathrm{mmHg} \mathrm{s} \mathrm{ml}^{-1}\right]}\end{array}$ & $\begin{array}{c}\text { Compliance } \\
{\left[\mathrm{ml} \mathrm{mmHg}^{-1}\right]}\end{array}$ \\
\hline $\mathrm{R}_{1,1}=0.8$ & $\mathrm{R}_{1,2}=508.0$ & $\mathrm{C}_{1,1}=0.01$ \\
\hline $\mathrm{R}_{1,3}=0.8$ & $\mathrm{R}_{1,4}=698.0$ & $\mathrm{C}_{1,2}=0.01$ \\
\hline $\mathrm{R}_{1,5}=0.6$ & $\mathrm{R}_{1,6}=156.0$ & $\mathrm{C}_{1,3}=0.02$ \\
\hline $\mathrm{R}_{1,7}=0.5$ & $\mathrm{R}_{1,8}=19.0$ & $\mathrm{C}_{1,4}=0.02$ \\
\hline $\mathrm{R}_{2,1}=0.5$ & $\mathrm{R}_{2,2}=188.5$ & $\mathrm{C}_{2,1}=0.05$ \\
\hline $\mathrm{R}_{2,3}=1.0$ & $\mathrm{R}_{2,4}=13.5$ & $\mathrm{C}_{2,2}=0.09$ \\
\hline $\mathrm{R}_{3,1}=2.0$ & $\mathrm{R}_{3,2}=160.0$ & $\mathrm{C}_{3,1}=0.25$ \\
\hline $\mathrm{R}_{3,3}=1.3$ & $\mathrm{R}_{3,4}=12.0$ & $\mathrm{C}_{3,2}=0.50$ \\
\hline \hline
\end{tabular}

Table 2: area-averaged values of velocity over cut planes at the proximal anastomosis, midgraft and distal anastomosis. The differences reported are between the $R$ and PO-A and PONA cases. The data below refers to Patient 2 (see Supplementary Data for Patients 1 and 3).

\begin{tabular}{cccc}
\hline & $\mathbf{R}$ & PO-A & PO-NA \\
\hline Proximal & 0.168 & 0.180 & 0.178 \\
\% difference & & $7 \%$ & $6 \%$ \\
\hline Mid plane & 0.647 & 0.683 & 0.672 \\
\% difference & & $5 \%$ & $4 \%$ \\
\hline Distal plane & 0.247 & 0.245 & 0.246 \\
\% difference & & $0.9 \%$ & $0.3 \%$ \\
\hline \hline
\end{tabular}


Table 3: values of percentage occlusion summarised for each patient.

\begin{tabular}{cllll}
\hline \hline \multirow{2}{*}{ Patient } & \multirow{2}{*}{ Location } & \multicolumn{3}{c}{ Simulated occlusion } \\
\cline { 3 - 5 } & & R case & PO-A case & PO-NA case \\
\hline \multirow{2}{*}{1} & Proximal & $58 \%$ & $58 \%$ & $58 \%$ \\
\cline { 2 - 5 } & Distal & $49 \%$ & $50 \%$ & $50 \%$ \\
\hline \multirow{2}{*}{2} & Proximal & $59 \%$ & $56 \%$ & $56 \%$ \\
\cline { 2 - 5 } & Distal & $65 \%$ & $65 \%$ & $65 \%$ \\
\hline \multirow{2}{*}{3} & Proximal & $38 \%$ & $31 \%$ & $32 \%$ \\
\cline { 2 - 5 } & Distal & $56 \%$ & $58 \%$ & $58 \%$ \\
\hline
\end{tabular}

